Military Technical College

Kobry El-Kobbah, Cairo, Egypt.

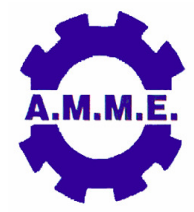

$15^{\text {th }}$ International Conference on Applied Mechanics and Mechanical Engineering.

\title{
DESIGN AND IMPLEMNTATION OF HYDRAULIC AUTO-LEVELING CONTROL SYSTEM FOR VEHICLE-BORNE PLATFORM BASED ON PLC
}

\author{
H. El-Gohary*, E. A. El-Kady** and M. E. Elrefaie **
}

\begin{abstract}
This paper puts forward hydraulic auto-leveling control system for vehicle-borne platform with four legs based on Programmable Logic Controller. In particular, a Siemens S7-300 (Programmable Logic Controller) PLC, and the accessional hydraulic circuit and control circuit is used. By introducing the theory of auto-leveling, the methods to design the hardware and software of the embedded control system are presented. The system adopts highly accurate two-axis tilt sensor and proportional direction control valves, optimizing auto-leveling strategy. The hardware and software of the embedded system make use of the concept of minimizing the error as possible on tilting axis corresponding to the required accuracy. The tests prove that this auto-leveling control system improves leveling-precision, performance stability of the system, shortens the leveling-time, and satisfies the maneuverability and operating requirements of the vehicle-borne platform in formidable nature conditions.
\end{abstract}

\section{KEY WORDS}

Auto-leveling, PLC, Platform, Vehicle-borne, Embedded system.

\footnotetext{
* Graduate Student, Mech. Eng. Dept., Faculty of Eng., Al-Azhar Univ., Cairo, Egypt.

** Professor, Mech. Eng. Dept., Faculty of Eng., Al-Azhar Univ., Cairo, Egypt.
} 


\section{NOMENCLATURE}

\section{List of Symbols}

$\alpha \quad$ Angle of rotation around $X$-axis.

$\beta \quad$ Angle of rotation around $Y$-axis.

\section{Abbreviation}

$\begin{array}{ll}\text { DCV } & \text { Proportional Directional Control Valve. } \\ \text { PT } & \text { Pressure Transducer. } \\ \text { DS } & \text { Displacement Sensor. } \\ \text { HJ } & \text { Hydraulic Jack. } \\ \text { SBG2 } & \text { Two axis tilt sensor. } \\ \text { HMI } & \text { Human Machine Interface SIMATIC TP 177A 6". } \\ \text { MCU } & \text { Main Control Unit. }\end{array}$

\section{INTRODUCTION}

Manually or automatically leveling system is usually used in vehicle born platform. Its function is to adjust the levelness of platform in specified time to satisfy required accuracy, so as to meet the normal working conditions of the vehicle-borne platform.

The manual leveling method was generally adopted in the past, by which the operator adjusted a screw again and again through observing the bulb of level. This method is time-consuming and the leveling precision is not high. In Recent years, the automatically leveling methods have been developed very quickly, including hydraulic leveling and mechatronic leveling systems. The hydraulic leveling has many advantages compared with the manual leveling, which include heavy load, higher precision and shorter time [1].

Because the adjusting time (the time needed to adjust the platform to satisfy the required accuracy) of traditional manual-leveling or auto leveling system is usually longer than five minutes, and their leveling accuracy of the platform is usually hard to reach 2 minute of angle, as well as their operating characteristic is susceptible to wild field and complex environment [2], hydraulic auto-leveling system with high accuracy is becoming the trend of the development.

In this paper, a control system for hydraulic auto-leveling system with four hydraulic jacks based on Programmable Logic Controller is introduced. Siemens S7-300 PLC (Programmable Logic Controller) and its accessional circuits is a smart and reliable device and is recently used widely. By using Siemens S7-300 as main control unit (MCU) Fig. 1, [3] , adopting highly accurate two-axis tilt sensor SB2G (RIEKER) Fig. 2, [4], digital pressure transducer and proximity switch as sensor, proportional directional control valves Fig. 3, [5] and hydraulic jacks as an actuators, an embedded auto-leveling control system is designed.

The hardware and software of the embedded system make use of the concept of error reduction on tilt axis to be as small as possible in accordance to the required 
accuracy. The tests prove that this auto-leveling hydraulic control system can satisfy the performance indexes and operating requirements of vehicle-borne platform.

\section{THEORY OF AUTO-LEVELING CONTROL SYSTEM}

\section{Legs Layout Projects}

The number of legs that sustain a platform may be three, four or six [6]. Figure 4 shows a project that uses Three - legged platform. Because a platform can be decided by three points or two intersecting lines, when the two intersecting lines are leveled, we can conclude that the platform is leveled. Two sensors for measuring tilting angles $\Theta 1$ and $\Theta 2$ are laid in two crossed direction to detect relative height of the points $(A, B$, and $C)$. When the three points are at the same height, the platform is leveled completely. The structure of this platform is simple and there is none statically indeterminate structure in it, because three points can only decide platform. However, the platform cannot carry big load because the number of legs is too small, and the ability that resists overturn is very limited. When encountering a big wind, the platform cannot maintain leveled and it is very difficult to adjust level, so the threelegged platform is only used for some simple and unimportant platform.

Figure 5 shows a four- legged platform. Two sensors for measuring $\alpha$ and $\beta$ which are tilting angles around $y$ - axis and $x$-axis sequentially are placed in two crossed direction, which can detect relative height of four points $A, B, C$ and $D$. This fourlegged platform can carry large load and can resist big overturn force, so it is widely used. However, three legs of the four- legged platform can sustain it if one leg is hanging in air and doesn't arrive to the ground as they are statically indeterminate. The problem must be solved when designing a four-legged platform.

The platform with six legs or more will increase statically indeterminate degree, which makes the design and calculation very complex. So, it is used only when load is very large and the other projects cannot meet with the need [1]. In general, the load in the platform is less than 30t, so four-legged platform is chosen in this paper when comparing with the other projects.

\section{Method and Process of Leveling}

In the following, two methods for adjusting the level platform level are described.

\section{a) One-direction adjusting}

First adjust direction $\mathrm{X}$, then adjust direction $\mathrm{Y}$, or in reverse turn. As for this method, the actions of legs are harmonious, but it takes too much time.

\section{b) Multi-direction adjusting}

The all legs act at the same time and arrive at the same given height. The adjusting speed by this method is high, but the control arithmetic is very complex because the speed and displacement of every leg is not equal and actions are restricted reciprocally. Moreover, the overload can happen because every leg bears the different force. So the method of one direction adjusting is used for the four-legged platform in this paper. 
The process of adjusting platform level degree is to adjust relative height of four points, so there are three methods for leveling as follows:

- The highest point immobility leveling method. The highest point does not move, but the other points rise to the same height to the highest point.

- The lowest point immobility leveling method. Let the lowest pointer stay fixed, but the higher points fall to the same height of the lowest point.

- The middle point immobility leveling method. The middle point of the platform stays fixed, and then the higher points fall and the lower points rise until all points are in the same level.

- As far as the efficiency is considered, the third method is the best [1], but it may produce one virtual leg, i.e. one leg does not carry load or hangs in the air. The second method will bring on the similar problems.

- When the first method is used the move direction of every leg is the same and so the precision of the leveling is higher.

- When the four legs all reach the ground and carry load, the process of leveling begins.

- At first, the highest point is concluded by the sensor's value. As shown in Figs. $6 \mathrm{a}$ and $6 \mathrm{~b}$, the sensors are placed in $\mathrm{X}$ and $\mathrm{Y}$ direction.

According to the value of $X$ and $Y$, the highest point can be concluded as follows [7]:

$X>0, Y<0$. The $A$ is the highest.

$X<0, Y<0$. The $B$ is the highest.

$X<0, Y>0$. The $C$ is the highest.

$X>0, Y>0$. The $D$ is the highest.

For example, the point $A$ is the highest. According to the signal of the horizontal sensors, the $X$ direction and $Y$ direction can be adjusted in turn. When the $X$ direction is adjusted at first, the process of leveling is as follows:

- The legs $A$ and $D$ stay immovable, the legs $B$ and $C$ rise to a given height at the same time, as is shown in Fig. (6a).

- The displacement of the leg is decided by the value of the sensor in $X$ direction.

- $\quad$ After the $X$ direction is adjusted to a level, the $Y$ direction should be adjusted according to the value of the sensor in $Y$ direction, which is shown in Fig. (6b).

- $\quad$ The process and method are the same to the $X$ direction.

- When the $X$ and $Y$ directions are leveled at the same time, the platform is leveled completely.

\section{Another Approach for Process of Leveling}

To study a four points supporting, hydro mechanical auto-leveling system, the layout of four supporting hydraulic jacks is a symmetrical rounded, and the slope angle $\alpha$, overturn angle $\beta$ of the platform is acquired by a highly accurate two-axis tilt sensor. The schematic plan of the platform is depicted in Fig. 7. In this figure the horizontal coordinate system is $\mathrm{OX}_{0} \mathrm{Y}_{0}$, and the platform coordinate system is $\mathrm{OX}_{1} \mathrm{Y}_{1}$.

The transformation matrix for the systems is defined as: 


$$
\begin{aligned}
\operatorname{Rot}(x, \alpha) & =\left(\begin{array}{ccc}
1 & 0 & 0 \\
0 & \cos \alpha & -\sin \alpha \\
0 & \sin \alpha & \cos \alpha
\end{array}\right), \\
\operatorname{Rot}(y, \beta) & =\left(\begin{array}{ccc}
\cos \beta & 0 & \sin \beta \\
0 & 1 & 0 \\
-\sin \beta & 0 & \cos \beta
\end{array}\right) .
\end{aligned}
$$

And since

$$
{ }_{1}^{0} \mathrm{R}=\operatorname{Rot}(\mathrm{x}, \alpha) \operatorname{Rot}(\mathrm{y}, \beta)
$$

Obtaining

$$
{ }_{1}^{0} R=\left(\begin{array}{ccc}
\cos \beta & 0 & \sin \beta \\
\sin \alpha \sin \beta & \cos \alpha & -\sin \alpha \cos \beta \\
-\cos \alpha \sin \beta & \sin \alpha & \cos \alpha \cos \beta
\end{array}\right)
$$

Since $\alpha, \beta$ is small, the expression can be approximated as:

$$
{ }_{1}^{0} \mathrm{R}=\left(\begin{array}{ccc}
1 & 0 & \beta \\
0 & 1 & -\alpha \\
-\beta & \alpha & 1
\end{array}\right)
$$

Adjusting of the levelness of platform is realized by the movement of supporting legs in $Z$ direction. Assuming the coordinates of the four mechanical legs inOX $\mathrm{Y}_{1}$ is,

$$
{ }^{1} \mathrm{P}_{\mathrm{i}}=\left({ }^{1} \mathrm{X}_{\mathrm{i}},{ }^{1} \mathrm{Y}_{\mathrm{i}},{ }^{1} \mathrm{Z}_{\mathrm{i}}\right)
$$

and the coordinates of the four mechanical legs inOX $\mathrm{X}_{0} \mathrm{Y}_{0}$ is,

$$
{ }^{0} \mathrm{P}_{\mathrm{i}}=\left({ }^{0} \mathrm{X}_{\mathrm{i}},{ }^{0} \mathrm{Y}_{\mathrm{i}},{ }^{0} \mathrm{Z}_{\mathrm{i}}\right) \text {. }
$$

Since,

$$
{ }^{0} \mathrm{P}_{\mathrm{i}}={ }_{1}^{0} \mathrm{R}{ }^{1} \mathrm{P}_{\mathrm{i}}
$$

Namely

$$
\left[\begin{array}{lll}
{ }^{0} X & { }^{0} Y & { }^{0} Z
\end{array}\right]={ }_{0}^{1} R\left[\begin{array}{lll}
{ }^{1} X & { }^{1} Y & { }^{1} Z
\end{array}\right]
$$

The expression of ${ }^{0} Z_{i}$ becomes:

$$
{ }^{0} Z_{\mathrm{i}}=-\beta^{1} \mathrm{X}_{\mathrm{i}}+\alpha^{1} \mathrm{Y}_{\mathrm{i}}+{ }^{1} \mathrm{Z}_{\mathrm{i}}
$$

Obviously, in the coordinate system $O X_{1} Y_{1}$

$$
{ }^{1} Z_{i}=0
$$

Hence the expression of ${ }^{0} Z_{i}$ can be written as:

$$
{ }^{0} Z_{\mathrm{i}}=-\beta^{1} \mathrm{X}_{\mathrm{i}}+\alpha^{1} \mathrm{Y}_{\mathrm{i}}
$$


By calculating the value of ${ }^{0} \mathrm{Z}_{\mathrm{i}}$, the highest leg can be got. The schematic plan of four legs is shown in Fig. 8.

Assuming $\mathrm{i}=\mathrm{h}$ as the highest leg, namely ${ }^{0} \mathrm{Z}_{\mathrm{h}} \geq{ }^{0} \mathrm{Z}_{\mathrm{i}}$, the altitude difference between three low legs and the highest leg can be got for $i=1,2,3$ by:

$$
e_{i}={ }^{0} Z_{h}-{ }^{0} Z_{i}
$$

In the progress of auto-leveling, keeping the highest leg still, and the other legs move upward according the value of $\mathbf{e}_{\mathrm{i}}$.After the movement, the platform will be horizontal in theory [3].

\section{HARDWARE DESIGN OF THE HYDRAULIC LEVELING SYSTEM}

The hardware of the hydraulic leveling system based on PLC consists of hydraulic system and control unit as shown in Figures 11, 12 and 13.

\section{Hydraulic System}

The hydraulic system is shown in Fig. 9.

\section{Main Control Unit}

The main control unit is shown in Fig.10, and consists of:

- PLC

- Set of sensors (one duel-axis tilt sensor, four displacement sensor, four pressure transducer)

- Cables and wiring.

- Software

\section{Control Mode and Control System Diagram}

Compared with all kinds of leveling methods, the highest point immobility leveling method is adopted. There are two types of control for selection, i.e. open loop control system and closed loop control system. According to the open loop control, the distances from every point to the highest point are detected by sensors and then every lower point is lifted to the level in return. As for the closed loop control, when the lower points rise, the sensors detect the level error at the same time and input the value to the controller continually. The process of leveling ends when the leveling error value meets the required precision. When the open loop system is used, the platform must be completely rigid and every leg should be upright with the platform because of the deformation of the platform and the legs. So it is difficult to maintain the precision for the open loop system. The closed loop control is in fact a feedback control. During leveling, the level error of the platform is detected by the sensor and this value is fed back to the controller, so the controller can end the leveling process when the error reaches a given value. The closed loop is chosen in this work because it can assure better level precision compared with the open loop control. The control system is shown in Fig10 as a block diagram. 


\section{Hardware Design for the Control System}

The hardware of the control system includes controller, proportional valves, sensors, HMI Siemens SIMATIC S7 (for monitoring the response of the system) and controller area network. The controller receives the signal from the sensor, and then gives command to the actuator according to the given program. At the same time, the controller can transfer the signal to the upper control center box through a data cable. The PLC includes a CPU226XM, an extend module EM235, EM232, etc. The PLC in the control system is shown in Fig. 11. The actuator system consists of hydraulic jacks, proportional valves, as shown in Fig.12.

The proportional valves are controlled by analog signals, which have advantages of high speed and high precision. The counterbalance lock valve keeps the system stable for long time. The proportional valves receive command from controller and open or closed according to the state signal to the controller, so it is easy to realize closed loop control.

A high precision electronic inclinometer is chosen as the sensor, which can detect two directions level error in the platform and switch the error into electrical signal and transfer it to the controller Fig. 13, when the leg moves to an ultimate place, the power of proportional valves will be cut off. Also, there is SIMATIC TP 177A 6"(HMI) monitor, Fig. 14, for monitoring the jack response and tilting angle of the platform.

The Controller Area Network (CAN), which is a field bus, is adopted as the communication interface between upper control center and PLC, who has physical identities feature according to the RS224 and the standard of hardware stands to CAN 2.0 A protocol. The PLC can report the work condition of control system to the upper control center through CAN. The information includes the leveling state and jacks situation. The communication interface between the operation panel and the PLC is the special integrated interface of the PLC. The operator can input and revise the parameters through the interface which are shown in the operation panel SIMATIC TP 177A 6"(HMI). The interface is a RS 224 interface, whose software protocol conforms to the special protocol that is constituted by Siemens for the PLC S7.

\section{Software Design for the Leveling System}

After the hardware is chosen for the control system, the software should be design through using proper tool. The tool program software STEP 7-Micro/WIN, which is special for the Siemens PLC S7-300, is used to design the software for the leveling control system. In the following, the function of every part is described.

\section{General}

The control system controls the leveling of a rigid platform carried out by four hydraulic pistons at its four corners. Each piston is controlled by a proportional hydraulic valve that adjusts the piston extension and consequently the inclination of the platform. The basic idea is to use three pistons to level the platform keeping one fixed as reference i.e. Origin of the coordinate system. The control function is 
implemented in a Siemens S7-300 PLC that is programmed according to requirement.

\section{Main control variables}

The PLC reads the inclination of the platform by way of an angle sensor which provides the inclination angles from the gravitational level position of two mutually orthogonal plans which in our case represents two plane of the of the platform to be leveled. The inclination angles are output by the sensor as two analog voltage signals read by analog input cards in the PLC. The PLC controls the proportional valves, hence the piston, through analog output voltage signals interfaced by Rexroth VT valve amplifier modules. As mentioned above the control philosophy is based on selecting one of the pistons (reference piston) as origin of the coordinate system and control the three other to level the platform. To be flexible in all field situations the reference piston is selected every time leveling is initiated. When operator initiates the leveling sequence all other four pistons are commanded to extend up to a preset pressure for each jack at which pressure transducer sends a signal to PLC. Each piston extends independently until it reaches its corresponding pressure limit and waits for the other. When all pistons reach certain limit pressure (to overcome problem of one of the pistons is hanging on air) the PLC calculates the inclination and deduces the highest point and set it as a new reference set point, then start to execute leveling algorithm calculation to identify for each piston the required action. Fig. 15 shows the flow chart of Software Design for the Leveling System. Accordingly the three free pistons are controlled in a closed loop and leveling of the platform through specified tilting angles tolerances is considered complete.

\section{EXPERIMENTAL RESULT}

The auto-leveling system is tested in a 16t vehicle-borne platform. The test proves that the system has high dynamic performance (improving the load distribution on different legs and the stability in the leveling process) and the levelness of platform after auto-leveling is less than 2 minute of angle (usually the levelness of platform of electro-hydraulic leveling system is 3-4minute of angle). The leveling time is usually 58 seconds, shorter than a postulated 90 second design requirement. Besides that, the system can keep the change of the levelness of the platform in 1 minute of angle in 24 hours after auto-leveling.

Indication shows that the angle $\alpha$ which is the tilting angle around the $X$ - axis is equal to $0.06^{\circ}=3^{\prime} 36^{\prime \prime}$, and the angle $\beta$ which is the tilting around the $Y$ - direction is equal to $0.03^{\circ}=01^{\prime} 48^{\prime \prime}$.

In order to analyze leveling time, by monitoring the system behavior using the Service Master Diagnostic (Meter from Parker devise), which plots flow, pressure and temperature versus time. [See Fig. 16]

\section{CONCLUSION}

The auto-leveling control system based on PLC microcontroller improves two key 
performance indexes for vehicle-borne platform: leveling-precision and leveling-time. Design as well as manufacturing procedure, the control system takes the specific requirement for special product into account adequately, in order to ensure the reliability and stability of the control system in formidable nature conditions. The system has positive significance in improving the equipment quality and presently advanced status of vehicle-born platform in industry. The modularized software and hardware shares the character of well-portability, and the auto-leveling strategy and algorithm can be applied in four points supporting related to area of industrial engineering.

\section{ACKNOWLEDGMENT}

The author would like to thank YASER FAHMY Company, especially Eng. Emad, Eng. Michael and staff in the control work group in this company.

\section{REFERENCES}

[1] Yang H. and Li G., "Research on an automatically leveling control system for vehicle-borne platform with high accuracy", 2006 IEEE/ASME International Conference on Mechatronics and Embedded Systems and Applications (IEEE Cat. No. 06EX1439), 2006, p. 5 pp., 6 refs, pp. CD-ROM, ISBN: 0-7803-97215. Publisher: IEEE, Piscataway, NJ, USA.

[2] Lu C. S., Huang D., Jin Z. and Huang Y.," The research and design of autoleveling control system for vehicle-borne radar platform based on AVR", 2006 IEEE/ASME International Conference on Mechatronics and Embedded Systems and Applications (IEEE Cat. No. 06EX1439), 2006, p. 5 pp., 12refs, pp. CD-ROM, ISBN:0-7803-9721-5.Publisher: IEEE, Piscataway, NJ, USA.

[3] Siemens Catalog ST70.2005.

[4] RIECKER Incorporated SB2G (Duel axis Inclinometer sensor).

[5] Rexroth catalog RE 64282105.00.

[6] Sheng N. J. and Yujian Z., "Horizontal Adjustment of Radar Antenna Truck," Measurement \& control technology, vol. 13, no. 4, Ju.1994, pp. 36-39.

[7] Yi F. and Baiji C., "Control system of automatically leveling of vehicle-borne radar", J. Huaz hong Univ of Sci. \& Tech. (Nature Science Edition), vol. 32, no. 6 , Jun. 2004, pp. 65-68. 


\section{Figures:}

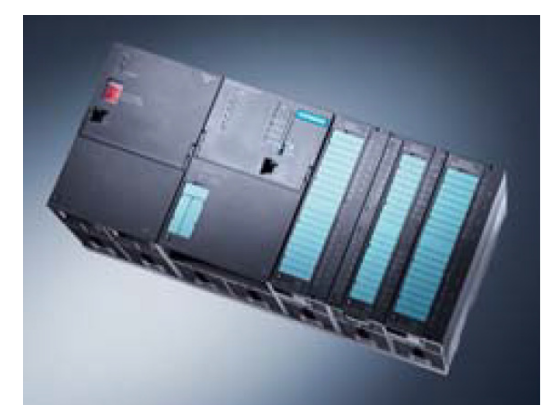

Fig. 1. Siemens S7-300 PLC.

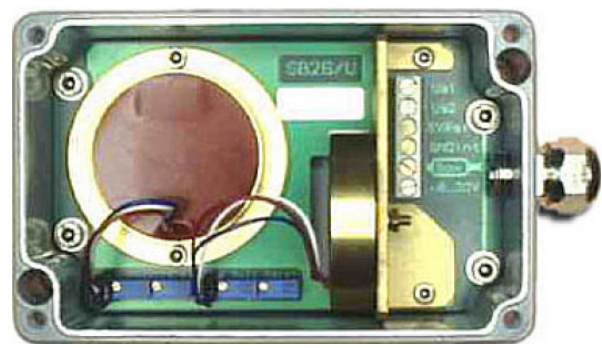

Fig. 2. SB2G (RIEKER).

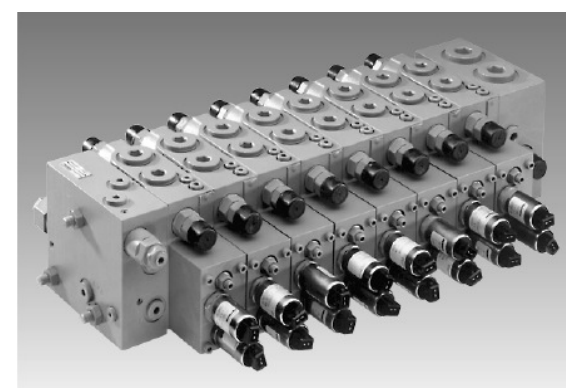

Control block type M4-15

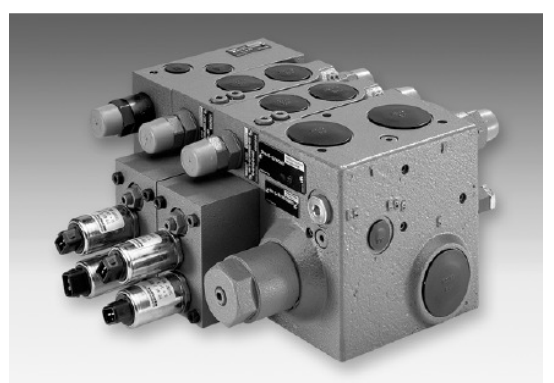

Control block type M4-15, inlet element with priority function

Fig. 3. High pressure load sensing control block of sandwich plate design Type M4-15.

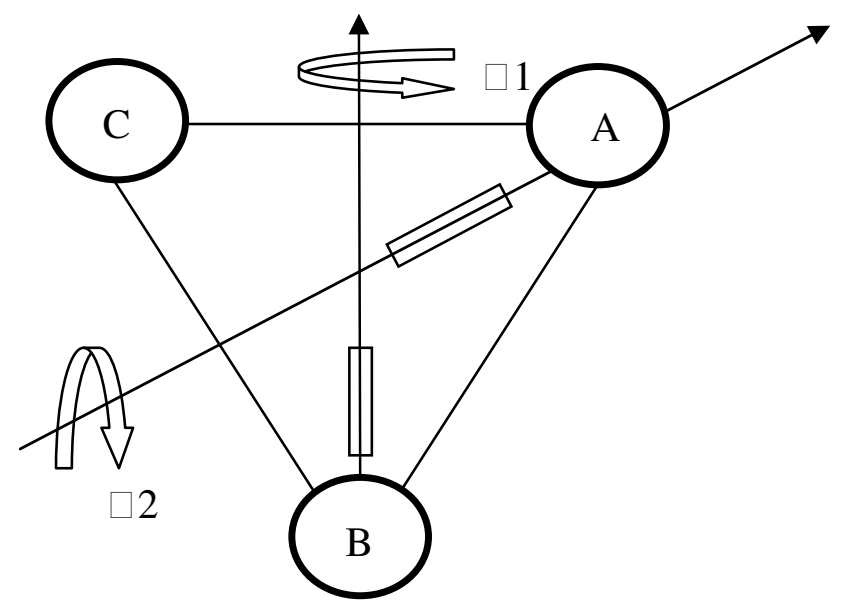

Fig.4. Three-legged platform. 


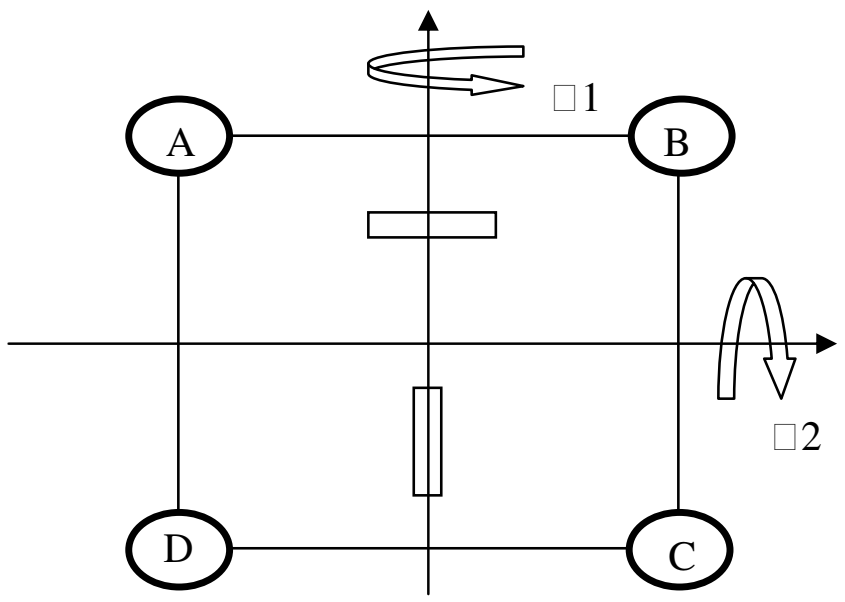

Fig. 5. Four- legged platform.

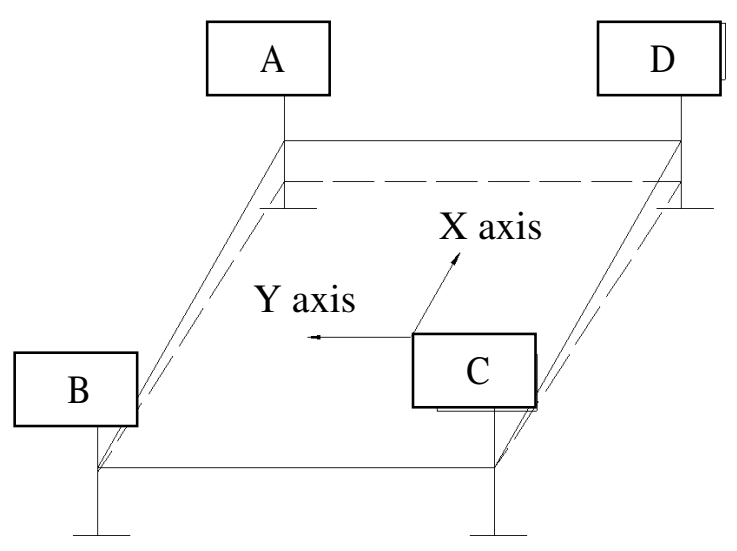

(a)

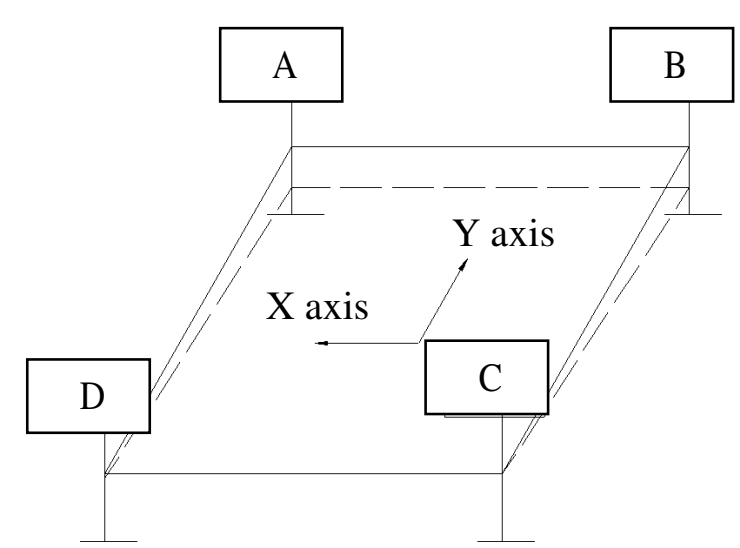

(b)

Fig. 6. Unleveled four-legged platform.

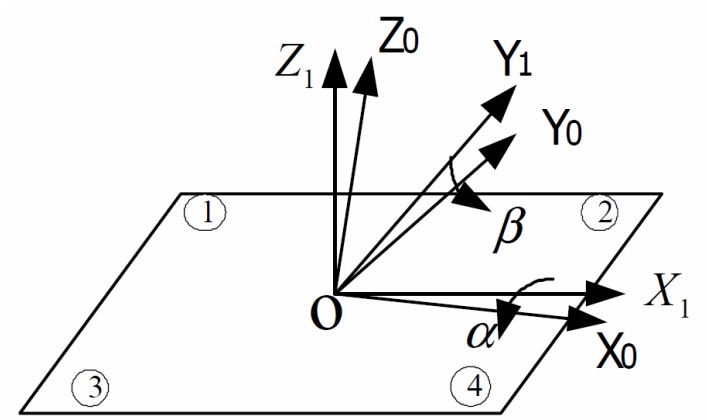

Fig. 7. The schematic plan of the four- legged platform. 


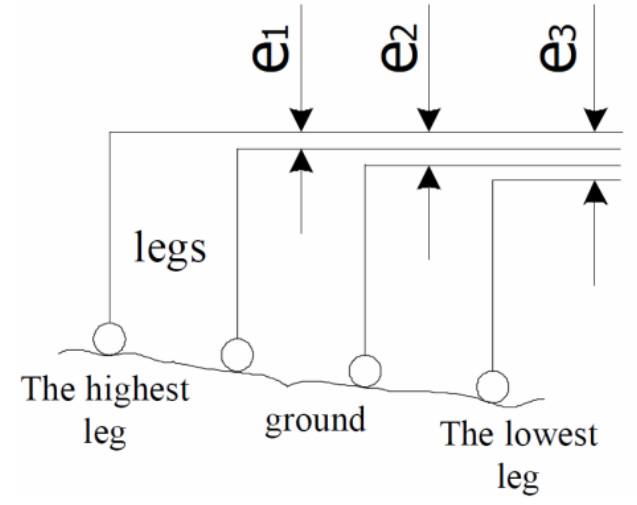

Fig. 8. The schematic plan of four legs.

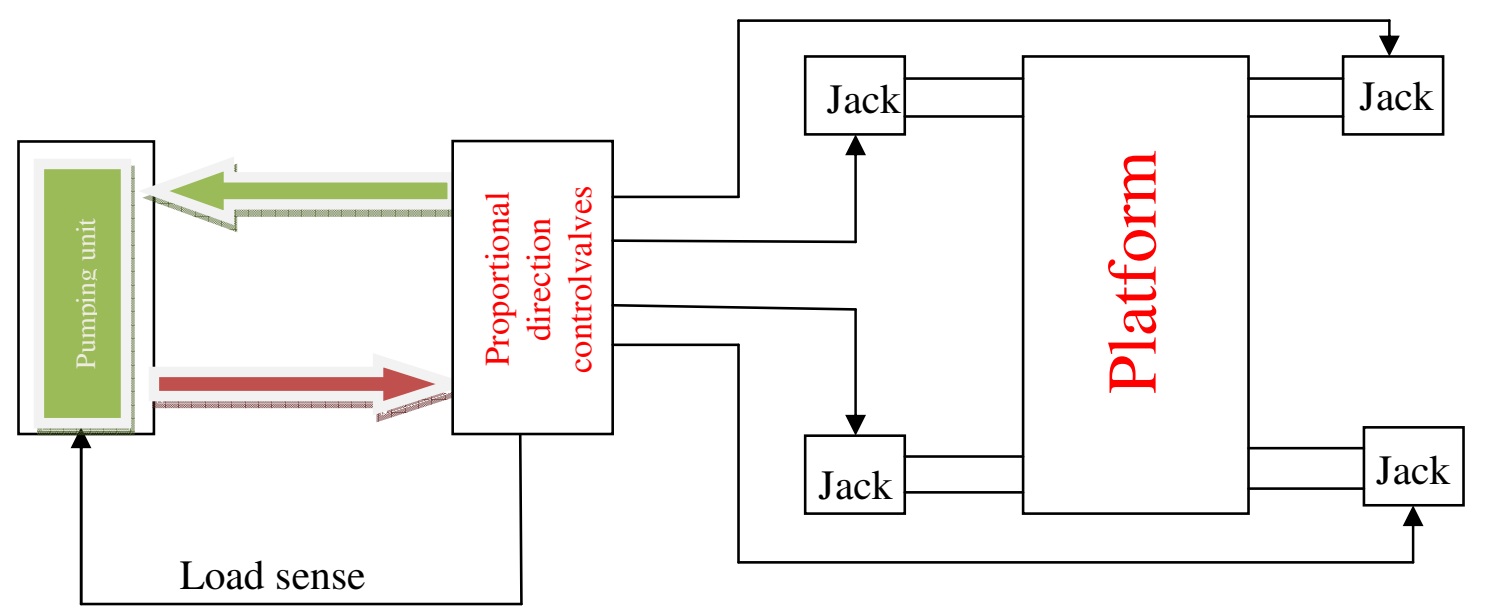

Fig .9. Block diagram of hydraulic system.

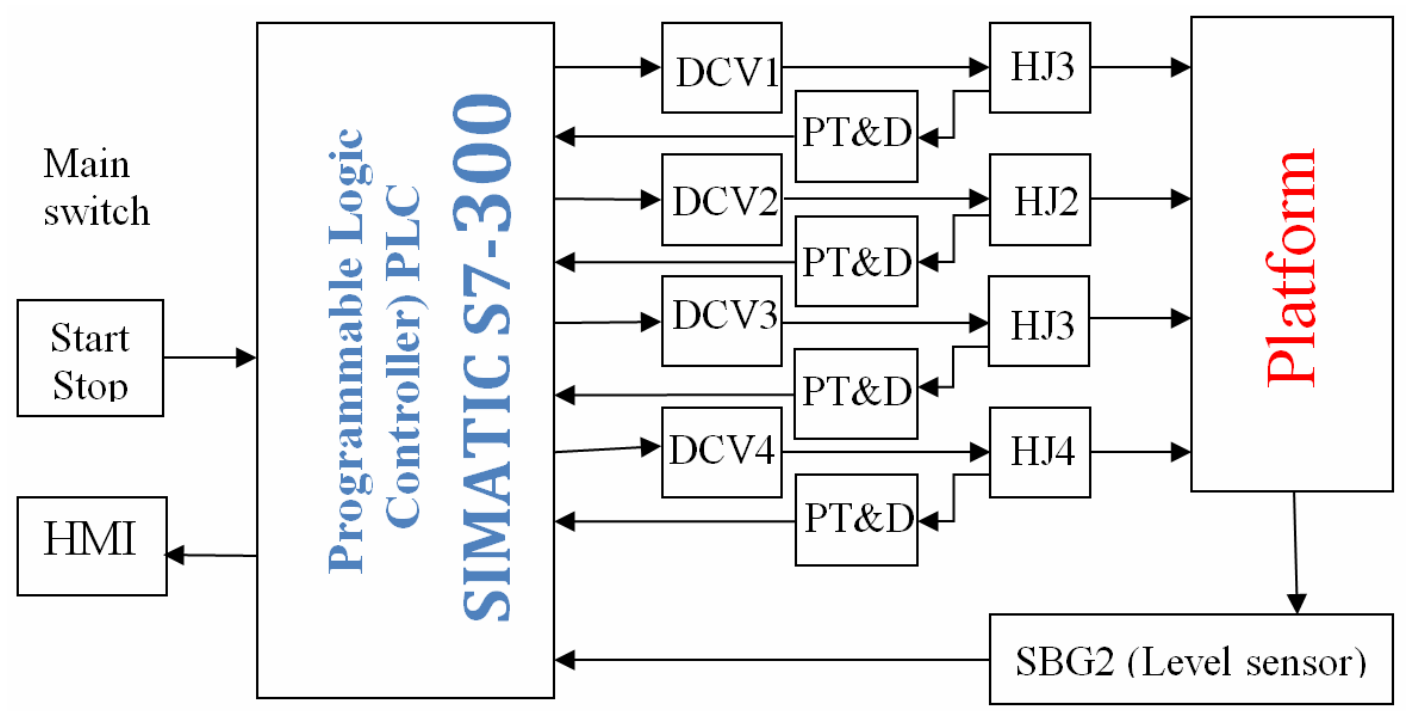

Fig . 10 block diagram of control system 


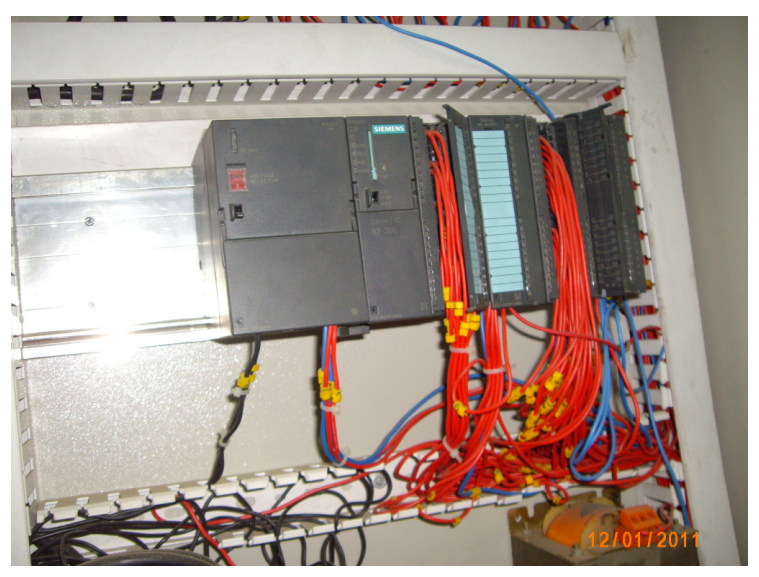

Fig. 11. PLC Control Panel.
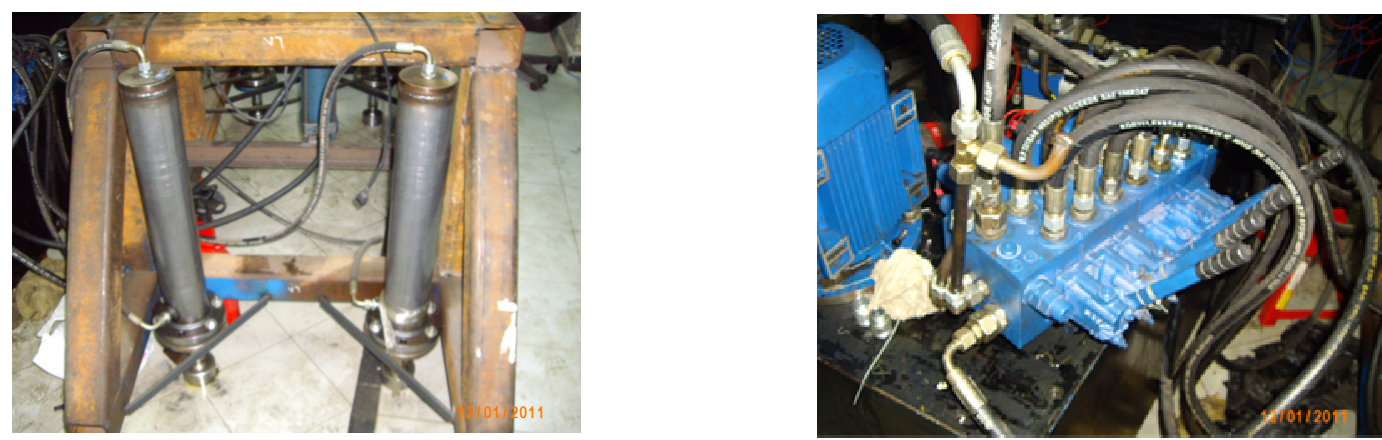

Fig.12. The structure of actuator system.

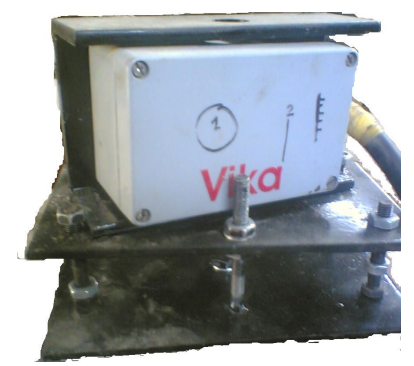

Fig. 13. SBG2 leveling sensor.

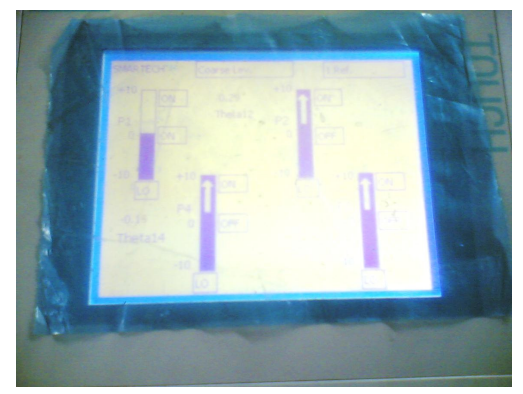

Fig.14. SIMATIC TP 177A 6"(HMI). 


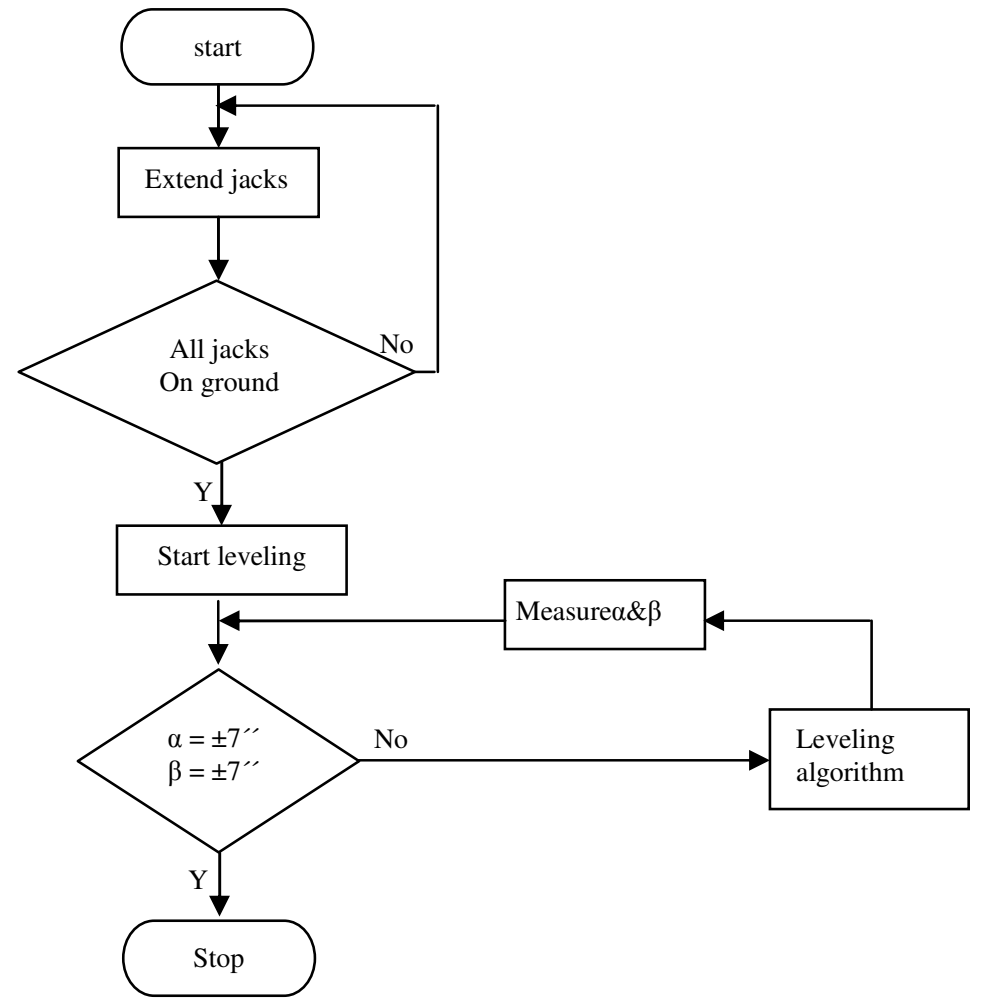

Fig. 15. Flowchart of Software Design for the Leveling System.

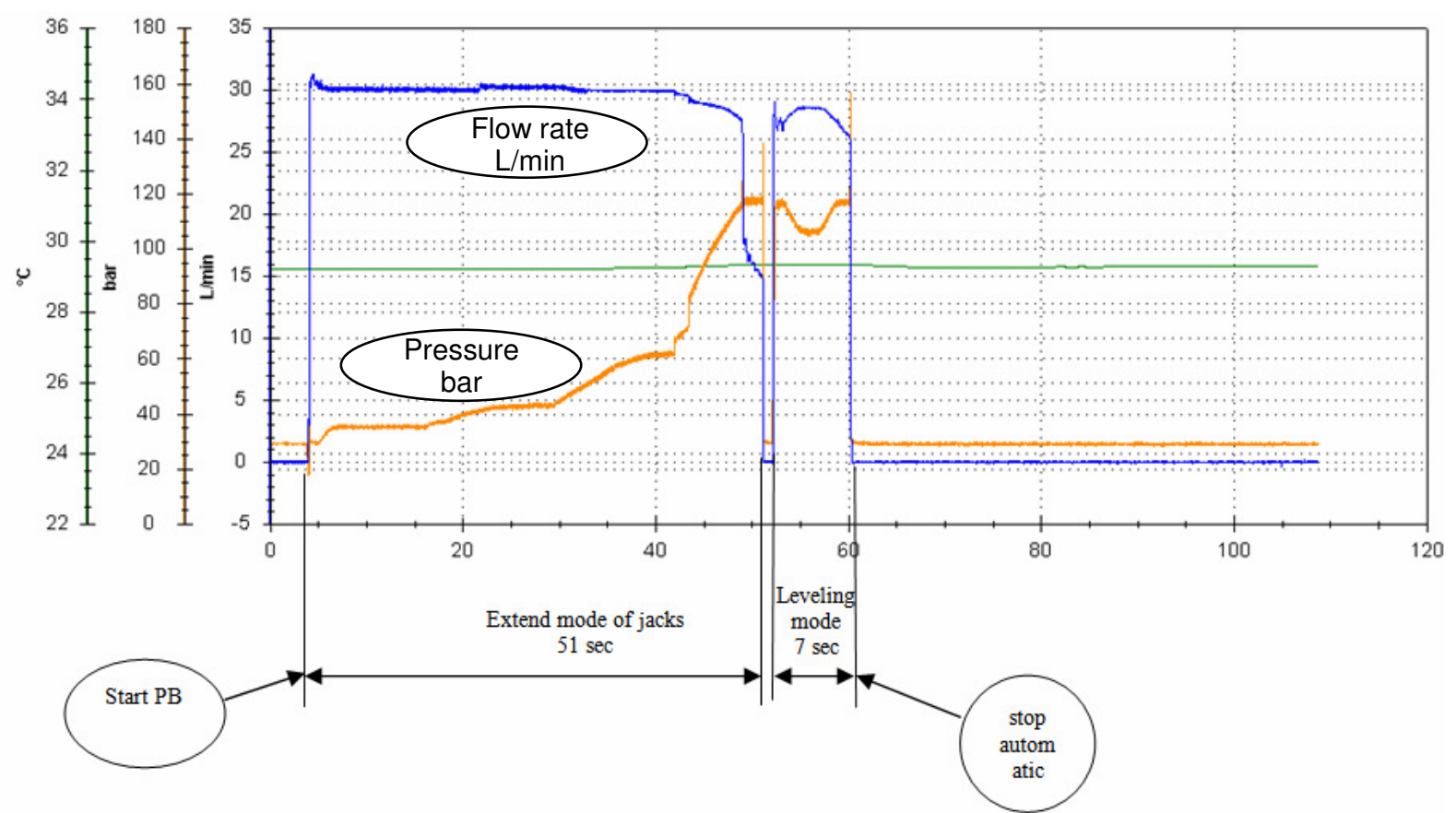

Fig.16. Leveling time analysis. 